\title{
Dynamic Restructuring of a Rhythmic Motor Program by a Single Mechanoreceptor Neuron in Lobster
}

\author{
Denis Combes, Pierre Meyrand, and John Simmers \\ Laboratoire de Neurobiologie des Réseaux, Université Bordeaux I and Centre National de la Recherche Scientifique, Unité \\ Mixte de Recherche 5816, 33405 Talence, France
}

\begin{abstract}
We have explored the synaptic and cellular mechanisms by which a single primary mechanosensory neuron, the anterior gastric receptor (AGR), reconfigures motor output of the gastric mill central pattern generator (CPG) in the stomatogastric nervous system (STNS) of the lobster Homarus gammarus. AGR is activated in vivo by contraction of the medial tooth protractor muscle gm1 and accesses the gastric CPG via excitation of two in-parallel interneurons, the excitatory commissural gastric (CG) and the inhibitory gastric inhibitor (GI). In the spontaneously active STNS in vitro, weak firing of AGR in time with gastric mill motoneurons (GM) reinforces an ongoing type I gastric mill rhythm in which all gastric teeth power-stroke motoneurons are synchronously active. With strong AGR firing, these phase relationships switch abruptly to a type II pattern in which lateral and medial teeth power-stroke motoneurons fire in
\end{abstract}

antiphase. Our results suggest that these bimodal actions on the gastric mill rhythm depend on the balance of firing of the $\mathrm{CG}$ and Gl interneurons and that selection of the pathway resides in their different postsynaptic sensitivities to AGR. Whereas high intrinsic firing rates of the CG neuron ensure that the excitatory pathway predominates during low levels of sensory input, strong synaptic facilitation in the Gl neuron favors the inhibitory pathway during high levels of receptor activity. Feedback from a single mechanosensory neuron is thus able, in an activitydependent manner, to specify different motor programs from a single central pattern-generating network.

Key words: Crustacea; Homarus gammarus; stomatogastric system; gastric mill motor network; mechanosensory neuron; sensorimotor integration; network reconfiguration; synaptic facilitation
From a variety of studies on both vertebrates and invertebrates, it is now clear that movement-related feedback from proprioceptors plays a crucial role in adapting the intensity and timing of rhythmic motor programs to changing behavioral demands. For example, phasic sensory signals can entrain and reinforce locomotor rhythms in lamprey (Grillner et al., 1981), crayfish (Sillar et al., 1986), cat (Andersson and Grillner, 1983), and locust (Reye and Pearson, 1988), thereby enabling central motor commands to match perceived movements. Furthermore, proprioceptive signals may play an important role in reinforcing an ongoing phase or initiating the transition between antagonistic phases of a movement cycle by positive and/or negative feedback reflexes (Pearson and Duysens, 1976; Bässler, 1986; Rossignol et al., 1988). For example, positive feedback from intraoral receptors allows us to bite with increasing strength on soft foods, but, with excessive closing forces, this input switches to negative feedback that inhibits jaw closing motoneurons (Sherrington, 1917).

In addition to transient adaptive adjustment of an ongoing motor rhythm, proprioceptive input can be involved in switching or selecting different patterns of motor output for different behavioral tasks. For example, in the mollusc Tritonia, light touch activates a central circuit, resulting in a withdrawal response, whereas stronger sensory stimuli induce the same circuit to produce escape swimming (Getting and Dekin, 1985). In Xenopus embryos, the same central motor circuitry can produce swimming

\footnotetext{
Received Oct. 19, 1998; revised Feb. 9, 1999; accepted Feb. 12, 1999.

This work was supported in part by the Human Frontier Science Program.

Correspondence should be addressed to Denis Combes, Laboratoire de Neurobiologie des Réseaux, Université Bordeaux I and Centre National de la Recherche Scientifique, Unité Mixte de Recherche 5816, Avenue des Facultés, 33405 Talence, France.

Copyright (C) 1999 Society for Neuroscience $\quad 0270-6474 / 99 / 193620-09 \$ 05.00 / 0$
}

or struggling behavior (Soffe, 1993), depending on the level of input from a single population of cutaneous receptors (Soffe, 1997). In both cases, however, the precise cellular pathways and mechanisms by which such sensory-induced switching occurs remain to be determined.

A suitable preparation for addressing this problem is the gastric mill rhythm-generating network in the stomatogastric nervous system (STNS) of the lobster Homarus gammarus. As a result of intensive study over the last two decades, the crustacean gastric mill motor network, including that of Homarus (Combes et al., 1999), is now well understood in terms of underlying cellular and synaptic mechanisms (Harris-Warrick et al., 1992). Moreover, a mechanoreceptor that provides movement-related feedback to the gastric mill central pattern generator (CPG) in Homarus has been identified (Simmers and Moulins, 1988a,b; Combes et al., 1995a). This sensor, the anterior gastric receptor (AGR), is particularly attractive for the cellular study of proprioceptive interactions with a cyclic motor program because it consists of a single large neuron whose signaling properties are known in detail. Moreover, as shown here, AGR accesses the lobster gastric mill network via two interneuronal pathways that have been identified previously (Combes et al., 1999).

Exploring mechanisms of sensorimotor integration is typically bedeviled by a conflict between the needs of experimental accessibility for electrophysiological recording, persistence of motor network activity, and conservation of proprioceptive feedback pathways (Rossignol et al., 1988). In our in vitro study, we were able to satisfy all three conditions by using rhythmically active, deafferented ("open-loop") STNS preparations in which the AGR-gastric mill network loop was artificially closed with appropriately timed experimental activation of the receptor neuron. 
A

AGR

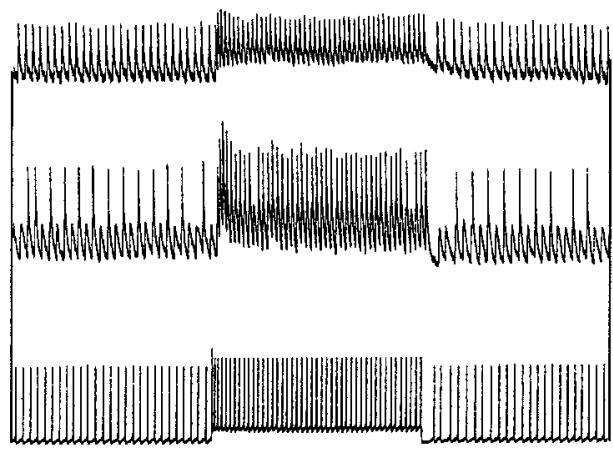

$\mathrm{I} 10 \mathrm{mV}$

$2 \mathrm{~s}$

B

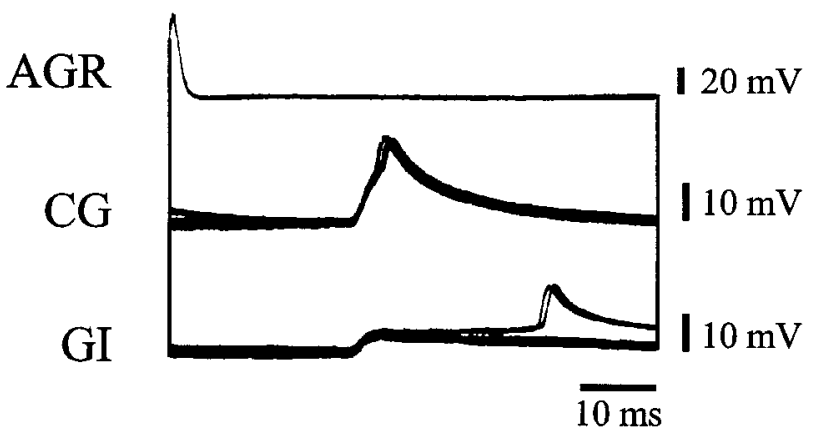

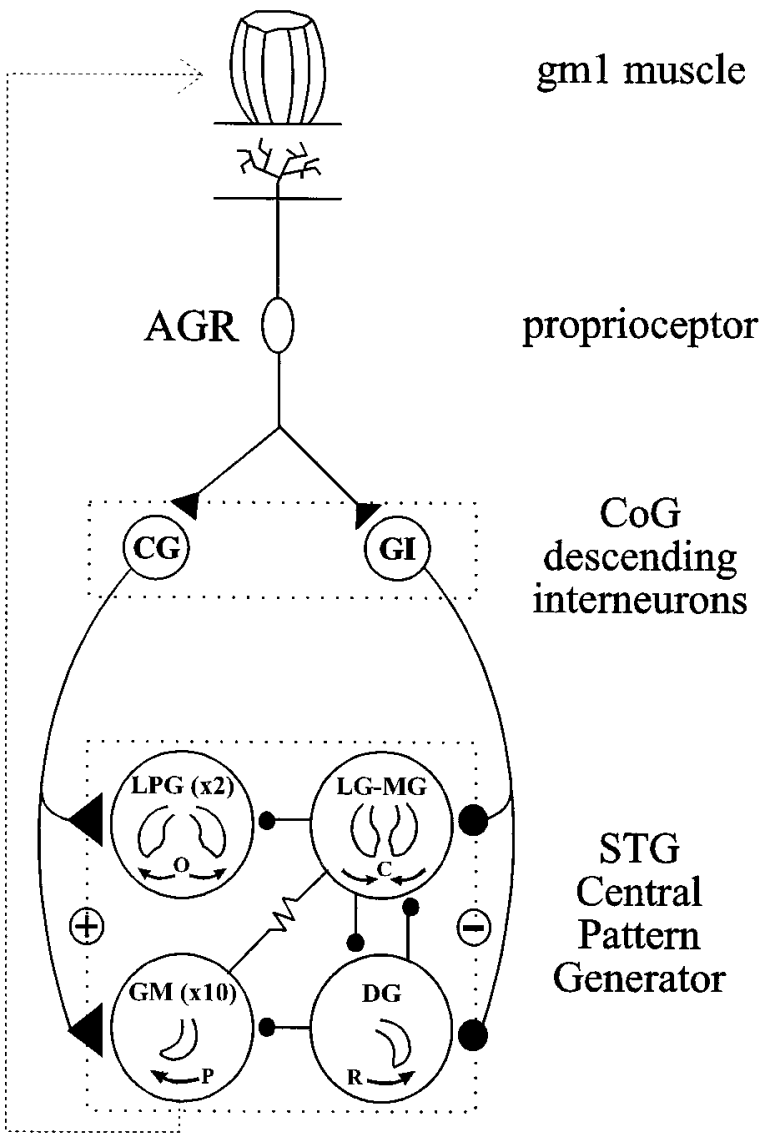

Figure 1. Cellular pathway through which lobster primary sensory neuron AGR influences the gastric mill CPG network (schema). The receptor, which arises from the tendon of the medial tooth protractor muscle gm1, projects to the STG via two descending CoG interneurons: the CG neuron, which excites the lateral teeth opener $(O)$ LPG and the medial tooth protractor $(P)$ GM motoneurons, and the GI neuron, which inhibits the lateral teeth closer $(C)$ LG-MG and medial tooth retractor $(R)$ DG motoneurons. Numbers of motoneurons of each subtype are indicated in the circles. Note that the single gastric mill network interneuron is not shown. $A$, Spontaneous and evoked AGR firing excites CG and GI interneurons. $B$, Each presynaptic AGR spike is correlated 1:1 and at constant latency with an EPSP in both CG and GI.

We show that AGR input, by acting through dual interneuronal pathways, has effects beyond simply reinforcing and adjusting ongoing gastric mill rhythmicity or assisting in the transition between cycle phases. Rather, according to the discharge of the receptor, AGR can evoke fundamental and persistent restructuring of the gastric mill rhythm to produce different activity patterns, similar to those spontaneously expressed in the intact animal.

A preliminary account of this work has been published previously (Combes et al., 1995b).

\section{MATERIALS AND METHODS}

All experiments were performed on in vitro preparations of the STNS of the lobster Homarus gammarus using dissection, electrophysiological, and data storage procedures as fully described in our accompanying paper (Combes et al., 1999).

Once isolated, the STNS was bathed in aerated saline maintained at $15-18^{\circ} \mathrm{C}$ and composed of (in $\mathrm{mM}$ ) $\mathrm{NaCl} 479.12, \mathrm{KCl} 12.74, \mathrm{CaCl}_{2}-2 \mathrm{H}_{2} \mathrm{O}$ 13.67, $\mathrm{MgSO}_{4} 10, \mathrm{Na}_{2} \mathrm{SO}_{4}$ 3.91, and HEPES 5, buffered to $\mathrm{pH} 7.45$. Under these conditions, the STNS generates robust spontaneous gastric mill rhythmicity, thereby allowing the study of AGR sensory input to an already active CPG.

Extracellular recordings were made with Vaseline-isolated platinum wire electrodes placed against appropriate motor nerve branches. Intracellular recording-stimulation was made with glass microelectrodes (tip resistance of 10-30 M 2 ) filled with $3 \mathrm{M} \mathrm{KCl}$. The commissural gastric (CG) and gastric inhibitor (GI) projection neurons were identified by their axonal projections in the superior oesophageal and stomatogastric nerves, according to their different postsynaptic effects on gastric mill motoneurons and on the basis of their synaptic responsiveness to AGR. AGR itself was stimulated by either intrasomatic depolarizing current injection or brief electrical shocks delivered via a platinum wire electrode placed on either of the two peripheral dendrites of the receptor.

In some experiments, the GI interneuron was selectively photoablated by intrasomatically injecting Lucifer yellow (3\% in distilled water; Sigma, Quentin Fallavier, France) and then illuminating the commissural ganglion $(\mathrm{CoG})$ for $30 \mathrm{~min}$ with intense blue light $(450-490 \mathrm{~nm})$. The gradual membrane depolarization of the GI neuron to zero indicated a successful photoinactivation (Miller and Selverston, 1979).

\section{RESULTS}

The gastric mill CPG network in the lobster stomatogastric ganglion (STG) consists of four motoneuron subsets, two of which control protraction [10 gastric motoneurons (GMs)] and retraction [one dorsal gastric (DG) motoneuron] of the gastric medial tooth, whereas the other two subsets drive opening [two lateral posterior gastric (LPG) motoneurons] and closing [the single lateral gastric (LG) and medial gastric (MG) motoneurons] of the lateral teeth (Combes et al., 1999) (Fig. 1, schema). As we described in our accompanying article (Combes et al., 1999), the gastric mill CPG receives two bilateral pairs of descending interneurons that originate in each $\mathrm{CoG}$ : the $\mathrm{CG}$ interneuron that monosynaptically excites the LPG and GM motoneurons and the GI interneuron that monosynaptically inhibits the LG-MG and DG motoneurons. It was shown previously that the AGR mech- 


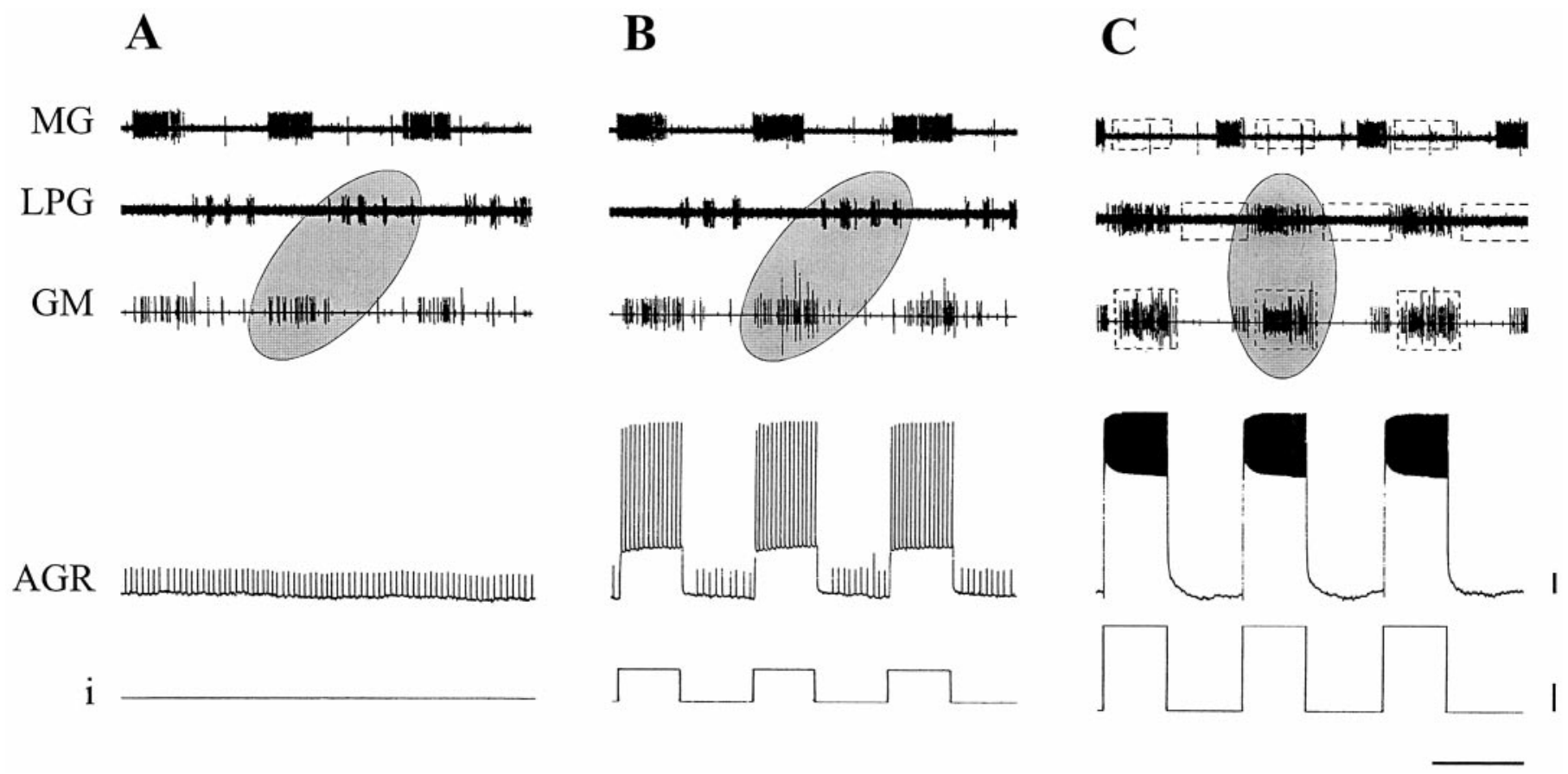

Figure 2. Multiple effects of AGR on the gastric mill CPG. Simultaneous extracellular recordings from three gastric mill motoneurons (MG, LPG, and $\mathrm{GM}$ ) and intrasomatic recording of AGR. A, Spontaneous in vitro gastric mill rhythm in the absence of AGR input. (The small depolarizing events in AGR are dendritic action potentials that do not generate axonal spikes.) In this pattern, the GM motoneurons fire in phase with the MG and out of phase with the LPG motoneurons. $B$, Cyclic depolarization of AGR by intracellular current injection $(i)$ causes it to fire axonal spikes weakly in time with GM bursts and increases GM neuron firing, with little other effect on gastric mill activity. $C$, Stronger AGR activation causes a switch in the phase relationships of the gastric mill pattern. Now, the GM motoneurons fire in phase with the LPG motoneurons. Dotted boxes indicate the pattern expressed in B. Calibration: vertical bars, $10 \mathrm{mV}, 2 \mathrm{nA}$; horizontal bar, $5 \mathrm{sec}$.

anoreceptor neuron arises from the tendon of the medial tooth protractor muscle and directly excites the two $\mathrm{CG}$ interneurons (Simmers and Moulins, 1988a,b) (Fig. 1A,B). We find here that AGR also excites the GI interneurons (Fig. $1 A$ ); this excitation is also probably direct, as indicated by the unitary fixed-latency EPSPs elicited by AGR impulses (Fig. $1 B$ ). Thus, with the STNS in vitro, an open-loop preparation is available in which a single primary mechanosensory neuron has access disynaptically to a motor pattern-generating network via two antagonistic interneuronal pathways (Fig. 1, schema).

To examine the role of sensory integration through these two projection pathways, we replaced the in vivo receptor activation by muscle contraction with direct intrasomatic stimulation of AGR in rhythmically active in vitro preparations. To reproduce such "closed-loop" conditions, AGR was stimulated in time with ongoing bursts in GM motoneurons, because it has been shown previously (Combes et al., 1995a) that AGR is activated by contraction of the muscle that these neurons innervate.

Figure 2 illustrates such an experiment in which simultaneous extracellular recordings from three motoneuron types (MG, LPG, and GM) of an already active gastric mill network before $(A)$ and during $(B, C)$ rhythmic GM-timed spike trains evoked in AGR. As was invariably seen in vitro during spontaneous gastric cycling (Combes et al., 1999) (Fig. 2A), the MG motoneuron bursts in antiphase with the LPG motoneurons but in phase with GM motoneurons. This coordination, which we refer to as the type I gastric mill pattern, corresponds to motor activity in vivo in which protraction of the medial tooth and closure of the lateral teeth would occur simultaneously. [Note that the depolarizing transients in the intrasomatic AGR recording in Fig. $2 A$ are spontaneously generated dendritic potentials that fail to trigger axonal action potentials (Combes et al., 1993).]

When AGR is cyclically depolarized to generate axonal spikes at a mean frequency of $<20 \mathrm{~Hz}$ in time with spontaneous GM motor bursts (Fig. 2B), the phase relationships between the different gastric mill motoneuron subsets remain unchanged, and relatively little change is seen in gastric mill activity, although GM neuron firing is enhanced. In contrast, rhythmic AGR stimulation that produces higher firing frequencies (in this experiment, $>20 \mathrm{~Hz}$ ) (Fig. $2 C$ ) causes a dramatic reorganization of the gastric mill pattern. In this new pattern, medial tooth GM motoneurons now fire in phase with lateral teeth LPG motoneurons rather than MG motoneurons (Fig. 2C, dotted boxes indicate type I pattern expressed in $B$ ). These phase relationships comprise a type II gastric mill pattern in which medial tooth protractor motor bursting is now in time with lateral teeth opener bursts.

As is seen in Figure 3, the switch between the two patterns occurs immediately after the onset of elevated AGR stimulation. In this experiment, a tonically autoactive AGR was recorded, along with the LPG, GM, and LG motoneurons. Note that the LG and MG motoneurons are strongly electrically coupled and therefore behave as a single functional entity (Selverston and Moulins, 1987; Combes et al., 1999). During spontaneous tonic AGR firing (Fig. 3, left), the typical type I gastric mill pattern (ellipse, alternate bursting in the LPG and GM neurons) is expressed. When AGR is rhythmically depolarized to step its mean firing rate from $\sim 5$ to $15-20 \mathrm{~Hz}$, the gastric mill pattern almost instantaneously switches to the type II pattern in which the LPG and GM neurons are now coordinately active in antiphase with the LG motoneuron. 


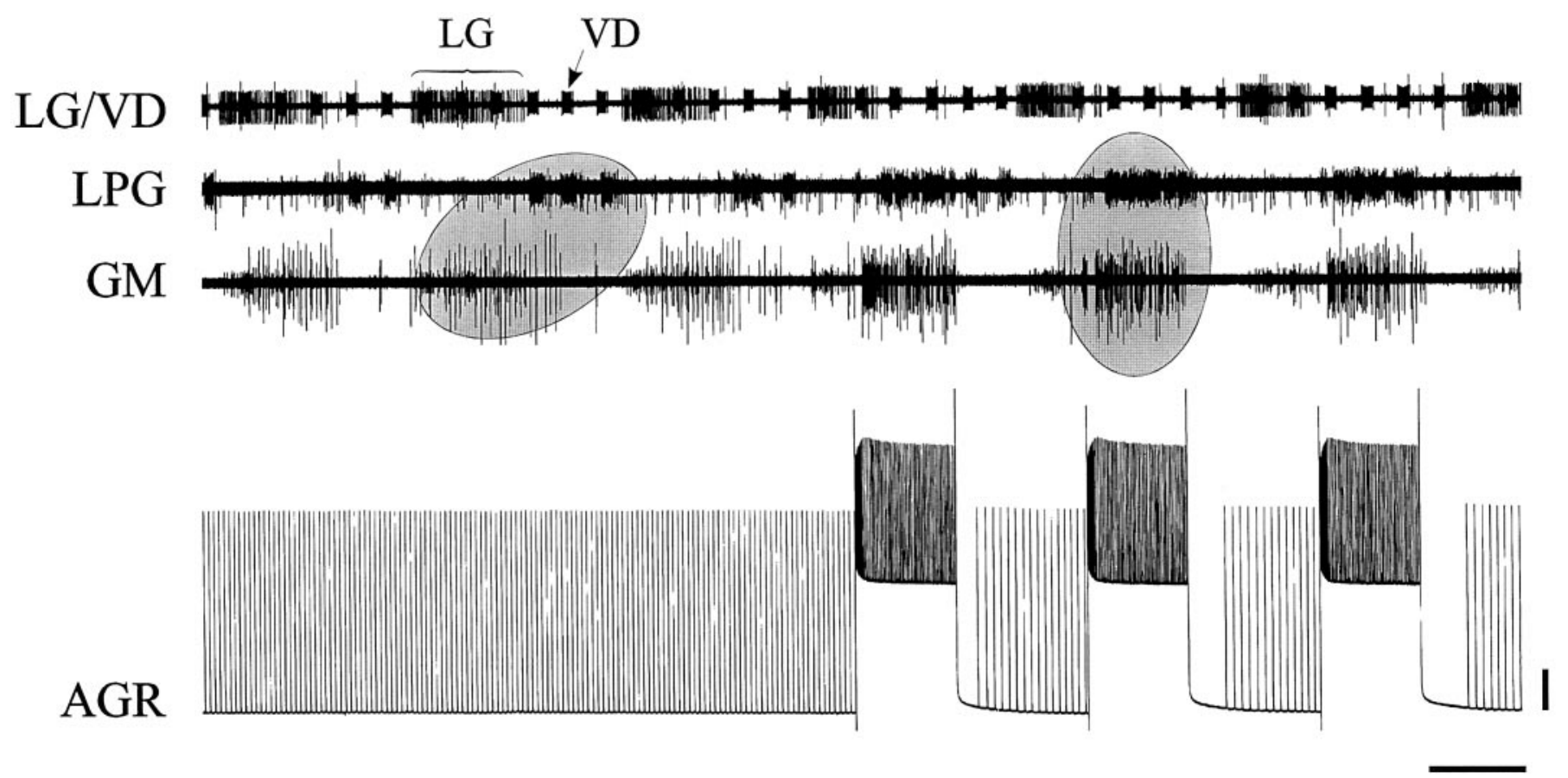

Figure 3. The transition between types I and II gastric mill patterns occurs immediately at the onset of AGR bursting (compare ellipses). The gastric mill CPG was monitored by extracellular recordings from the GM, LPG, and LG motoneurons. Note that the axon of the latter is in a nerve carrying the ventricular dilator $(V D)$ motoneuron axon of the pyloric network. Note also that AGR was spontaneously active in the absence of injected current. Calibration: vertical bar, $10 \mathrm{mV}$; horizontal bar, 4 sec.

The first conclusion from these series of experiments $(n=9)$ therefore is that, when AGR is activated in time with GM motoneuron bursts, depending on its firing frequency the receptor either reinforces the ongoing type I gastric mill pattern or rapidly reconfigures the output of the network into a type II gastric mill pattern.

\section{Role of descending sensory interneurons}

AGR has access to the gastric mill network via direct excitation of two in-parallel interneurons, the excitatory CG and inhibitory GI (Combes et al., 1999). Moreover, these two interneurons appear to be the only pathway by which the receptor reaches the gastric mill CPG. Simmers and Moulins (1988a) have already shown that AGR has no direct access to gastric mill neurons in the STG. In addition, the experiment shown in Figure 4 strongly suggests that the receptor is unable to influence the gastric mill network without the $\mathrm{CG}$ and GI neurons. Under control conditions in this experiment (Fig. 4A), extracellular AGR stimulation (shocks at $30 \mathrm{~Hz}$ for $3 \mathrm{sec}$ ) caused GM neuron excitation and MG neuron inhibition, as already seen in Figures 2 and 3. In Figure 4B, one $\mathrm{CoG}$ had been removed by dissection, and the CG neuron in the remaining CoG hyperpolarized. Moreover, the GI neuron in this CoG had been photoinactivated by Lucifer yellow injection and blue light illumination (see Materials and Methods). As a result of these treatments, spontaneous gastric mill rhythmicity had ceased. Under these conditions, AGR stimulation at even higher frequencies than those used in Figure $4 A$ had no effect on the two recorded gastric mill motoneurons.

Given that AGR appears to access the gastric mill network uniquely via the CG and GI neurons, it should be possible to mimic the effects of AGR input by direct manipulation of these two interneurons. One of three such experiments is illustrated in Figure 5 in which the CG and GI neurons were recorded intra-
A

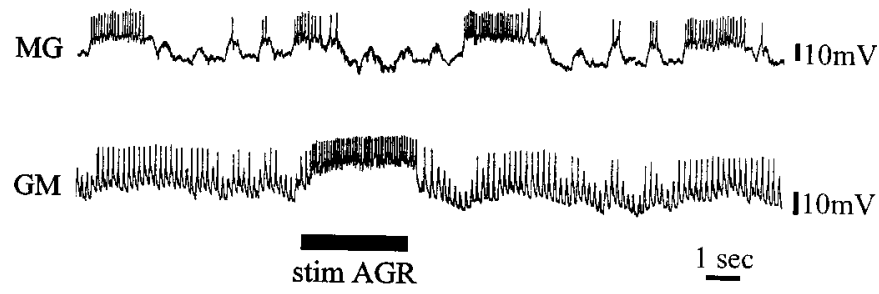

B

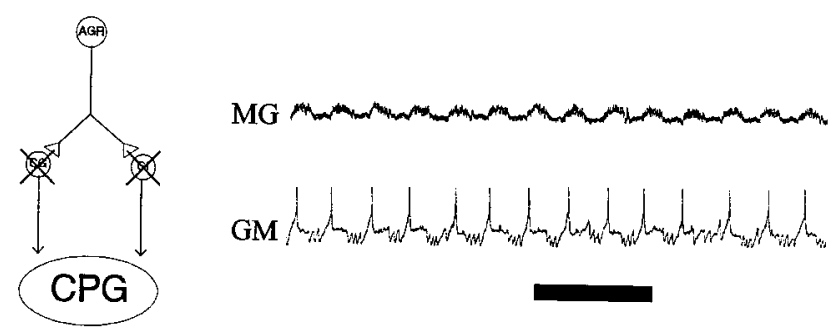

Figure 4. AGR projects to the gastric mill CPG only via the two descending CG and GI interneurons in each CoG. $A$, In control conditions, stimulation of AGR ( $30 \mathrm{~Hz}$ for $3 \mathrm{sec}$, horizontal bar) excites the GM motoneuron and inhibits the MG neuron. $B$, When the right CoG was removed and the two sensory interneurons in the left $\mathrm{CoG}$ were silenced (by hyperpolarizing CG and photoablating GI), AGR stimulation no longer affected the gastric mill motoneurons.

cellularly, along with extracellular recordings from motoneurons of the three major gastric mill subgroups. Figure $5 A$ shows spontaneous gastric mill cycling in the absence of phasic interneuronal discharge. However, periodic stimulation of the interneurons, either CG individually (Fig. $5 B$ ) or CG and GI simultaneously 


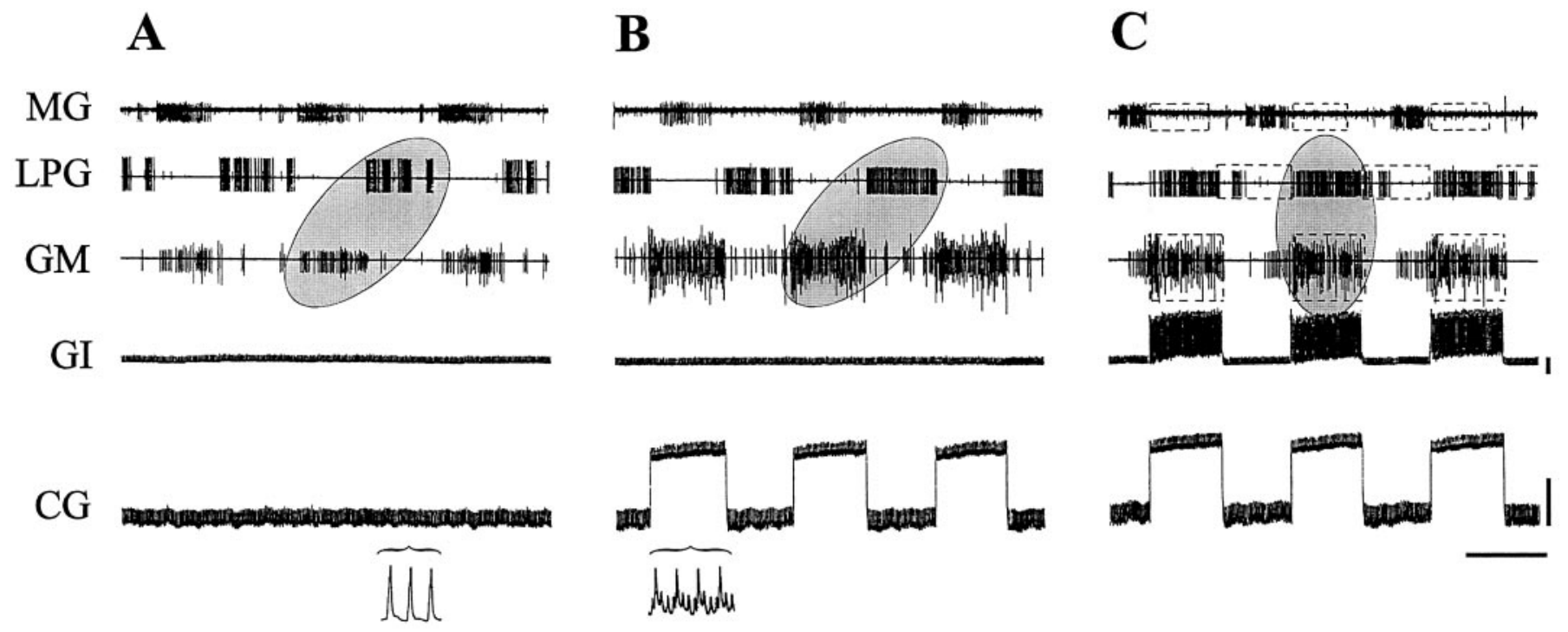

Figure 5. Direct effects of the CG and GI interneurons on the gastric mill network. $A$, Spontaneous gastric mill rhythm monitored by extracellular recordings of the MG, LPG, and GM motoneurons. The CG and GI interneurons were spontaneously silent (the depolarizing events in the CG neuron are EPSPs; see expanded time base recording in inset). B. Cyclic experimental activation of the CG neuron in GM neuron time reinforces the ongoing gastric mill pattern (the smaller depolarizing events in the CG neuron are action potentials; see inset). $C$, Simultaneous cyclic depolarizations of both interneurons reconfigure the gastric mill pattern. Dotted boxes represent the pattern expressed in B. Calibration: vertical bars, $20 \mathrm{mV}$; horizontal bar, $4 \mathrm{sec}$.

(Fig. 5C) by current injection in time with GM neuron firing, had effects on the rhythm identical to those produced by direct AGR stimulation (Fig. 2). Thus, activation of the CG neuron alone clearly enhanced (Fig. $5 B$ ) the ongoing type I pattern, whereas conjoint activation of the CG and GI neurons induced type II gastric mill activity (Fig. 5C) in which LPG and GM neurons fire conjointly. In this experiment, the evoked discharge of both the CG and GI neurons was similar $(\sim 50 \mathrm{~Hz})$, but we have found that phasic firing of the GI neuron as low as $20 \mathrm{~Hz}$ (in conjunction with simultaneous CG neuron activity) can induce the type II pattern.

Thus, like AGR stimulation at weak to moderate firing rates (Fig. $2 B$ ), activation of the CG neuron alone (Fig. 5C) is able to promote the ongoing type I gastric mill rhythm. Similarly, coactivation of the CG and GI interneurons (Fig. $5 B$ ) produces a type II gastric mill pattern that resembles the pattern AGR induces when firing strongly (Fig. 2C). Can AGR itself under some conditions preferentially activate the CG neuron and thereby reinforce the gastric mill rhythm, and under others simultaneously drive the CG and GI neurons to reconfigure the gastric mill pattern? This possibility is explored in the following experiments.

\section{Differential synaptic effects of AGR on the CG and GI neurons}

In a first step to assess the ability of the receptor to differentially activate the two projection pathways, we measured the synaptic responsiveness of the CG and GI interneurons to input from AGR in three experiments. In the experiment shown in Figure $6 A$, the GI and CG neurons were recorded simultaneously with an AGR whose firing frequency was manipulated by intrasomatic current injection (Fig. $6 A_{1}$ ). The mean \pm SEM evoked discharge frequencies of postsynaptic CG and GI were then expressed as a function of the firing rate of AGR (Fig. 6 $A_{2}$ ). At lower receptor discharge rates $(<20 \mathrm{~Hz})$, the response of the $C G$ interneuron was considerably higher (approximately three times) than that of the GI interneuron. For example, when AGR fired at $10 \mathrm{~Hz}$, the CG neuron fired at an average of $12 \mathrm{~Hz}$, whereas the GI neuron fired at a mean rate of only $2.5 \mathrm{~Hz}$. However, as AGR spike frequency increased, the discharge rate of the GI interneuron increased exponentially until its response curve approached and eventually crossed that of the CG interneuron. Thus, when AGR is firing at low frequencies, the CG interneuron is more active, but at high receptor firing rates, the discharge of the GI neuron increases relative to that of the CG interneuron so that eventually both interneurons become equally active. The different responsiveness of the two interneurons to AGR input is confirmed in Figure $6 B$ in which data from the three experiments analyzed were pooled and the mean \pm SEM interneuronal discharge rates (in each case expressed as a percentage of the firing rate when AGR itself was firing at $15 \mathrm{~Hz}$ ) are plotted as a function of imposed AGR discharge at 15, 20, 25, and $30 \mathrm{~Hz}$. In a strikingly similar manner in all three experiments, whereas CG neuron firing rose steadily by $\sim 75 \%$ in response to a doubling of spike frequency of AGR from 15 to $30 \mathrm{~Hz}$, GI neuron firing increased dramatically by $>500 \%$.

What mechanism could underlie these different sensitivities of the two interneurons to AGR input? To address this, we compared the mean \pm SEM steady-state amplitude of EPSPs evoked in the two interneurons at various AGR firing frequencies. (Note that EPSPs in both interneurons attained steady-state levels within $<1 \mathrm{sec}$ of all depolarizing current-induced changes in AGR firing rate.) As seen in the single experiment of Figure $7 \mathrm{~A}$ and the pooled data from the three experiments in Figure $7 B$, whereas EPSPs recorded in the GI neuron increase smoothly with stepwise increases in receptor firing rate, PSPs in the CG neuron decrease in amplitude. In the example of Figure $7 A$, GI neuron EPSPs increased from 10 to $30 \mathrm{mV}$, and CG neuron EPSPs decreased from 10 to $4 \mathrm{mV}$, as firing frequency of AGR increased from 8 to $30 \mathrm{~Hz}$. In other words, the synaptic response of GI to AGR is strongly facilitating, whereas that of CG appears to 

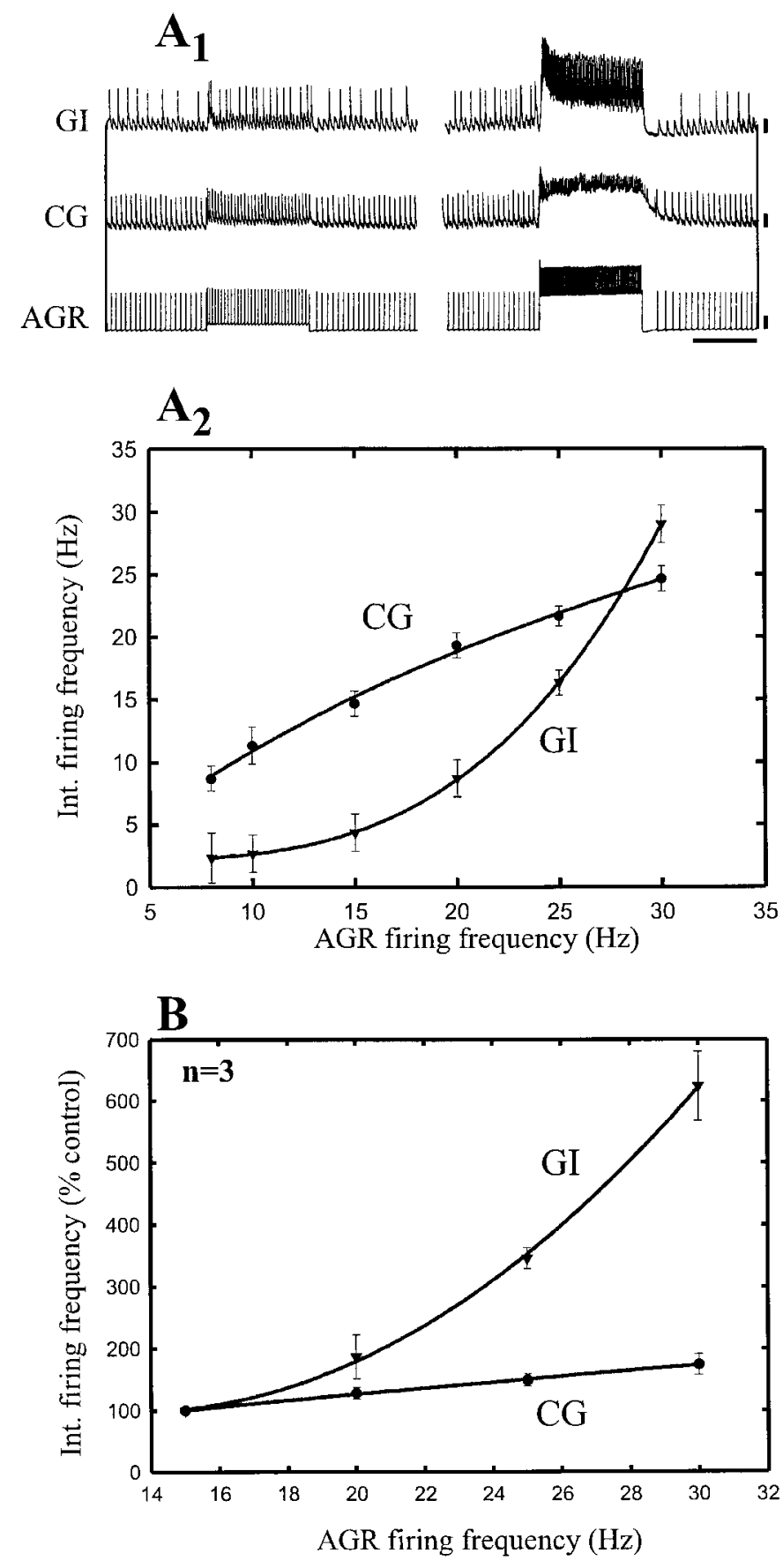

Figure 6. Differential sensitivities of the CG and GI interneurons to synaptic excitation by AGR. $A$, Single experiment. $A_{1}$, Simultaneous intracellularly recorded responses of the two interneurons to an evoked increase (by pulsed current injection) in AGR firing rate from a spontaneous mean of 5-10 (left) and 21 (right) Hz. Calibration: vertical bars, $10 \mathrm{mV}$; horizontal bar, 2 sec. $A_{2}$, Plots of CG and GI neuron responses to a range of AGR firing frequencies from 8 to $30 \mathrm{~Hz}$. Each point is the mean \pm SEM firing rate during at least three AGR stimulations. $B$, Pooled data from three experiments. Each point is the mean \pm SEM firing rate (relative to control rate when AGR fires at $15 \mathrm{~Hz}$ ) of the three corresponding interneurons in response to stepwise changes in AGR discharge as seen in $A_{l}$.

defacilitate. However, note that the individual CG and GI neuron EPSPs are superimposed on a depolarizing envelope because of temporal summation that was itself proportional to receptor firing rate (Fig. $6 A_{1}$ ). Thus, the apparent decrease in CG EPSP ampli-
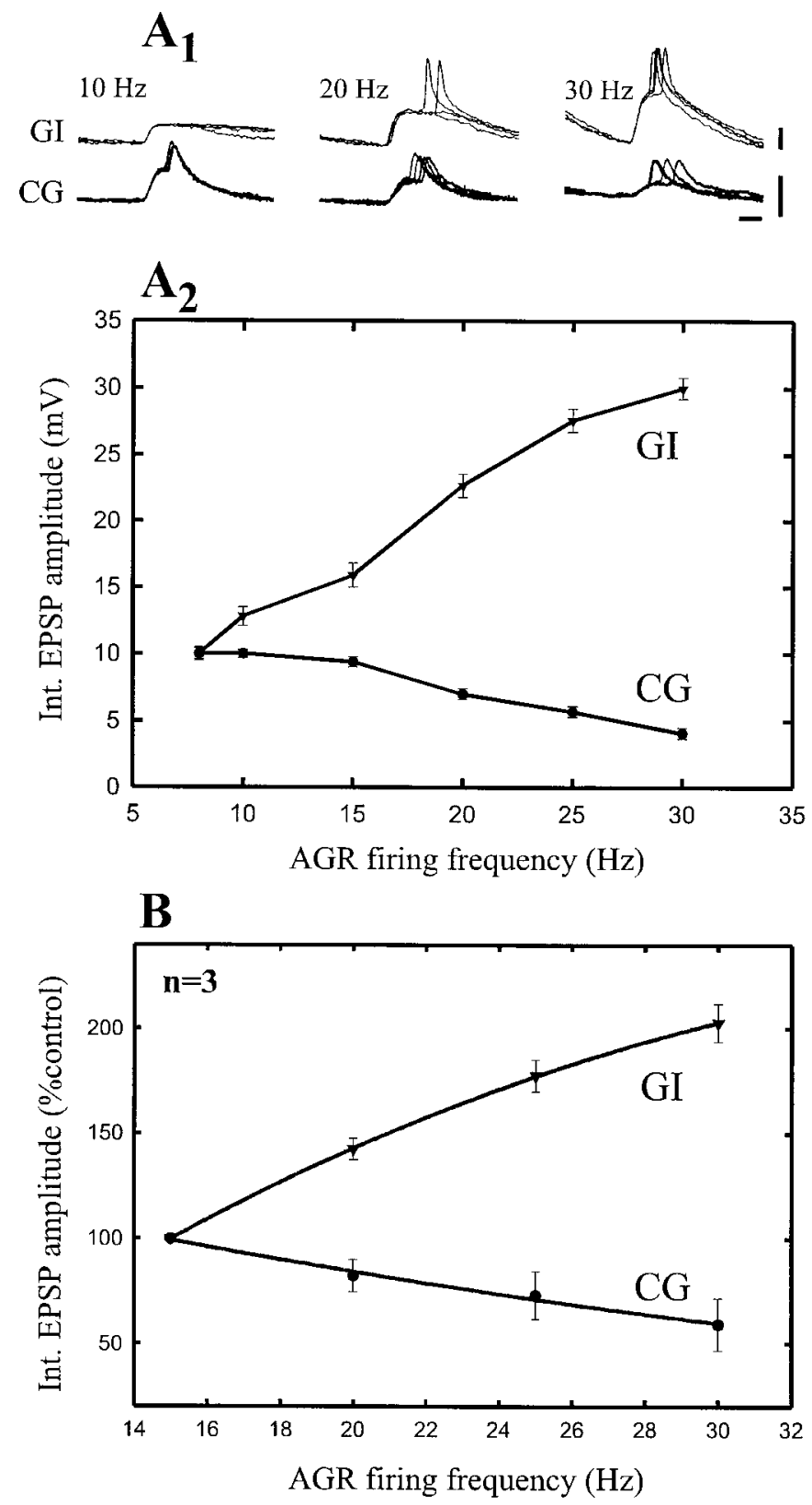

Figure 7. The GI, but not the CG, interneuron displays strong synaptic facilitation to AGR input. $A$, Single experiment (same as in Fig. $6 A_{1}$ ). $A_{1}$, Superimposed traces $(n=4)$ show AGR-evoked EPSPs (measured at steady-state levels) in the two interneurons at three different mean frequencies of receptor stimulation. Note strong increase in GI neuron EPSP amplitude, whereas CG neuron EPSPs decrease. Calibration: vertical bars, $10 \mathrm{mV}$; horizontal bar, $5 \mathrm{msec}$. $A_{2}$, Relationship between EPSP amplitude in the two interneurons over a range $(8-30 \mathrm{~Hz})$ of AGR firing frequencies. Each point is the mean \pm SEM amplitude of at least 10 synaptic events. $B$, Pooled data from the same three experiments as in Figure $6 B$. Each point is the mean \pm SEM steady-state amplitude (relative to control amplitude when AGR fires at $15 \mathrm{~Hz}$ ) of each interneuron in response to stepwise changes in receptor firing rate.

tude could be caused by the decrease in driving force resulting from this underlying depolarization rather than true defacilitation.

In conclusion, although a high spontaneous firing rate of the CG neuron results in its activity predominating during low to moderate levels of receptor input, strong synaptic facilitation in 
the GI neuron ensures that at higher levels of AGR activity it becomes relatively more effective and, in combination with the CG neuron, reconfigures gastric mill output into pattern II.

\section{DISCUSSION}

We have explored a sensorimotor system that consists of a limited number of neuronal elements, all of which have been identified electrophysiologically and their synaptic relationships established. This system (Combes et al., 1999) is comprised of a single primary mechanoreceptor neuron (AGR) that projects to the 16 neuron gastric mill CPG network via two intercalated interneurons, one excitatory ( $\mathrm{CG})$ and the other inhibitory $(\mathrm{GI})$. We have taken advantage of a further important feature of this preparation, namely the ability of the gastric mill CPG to remain spontaneously active in vitro in the absence of all sensory feedback. This enabled us to examine the specific effects of AGR on an already active gastric mill circuit in a manner that is generally impossible in other preparations. In many other preparations, the influence of sensory feedback on ongoing CPG activity has been necessarily restricted to semi-intact or intact preparations in which the central intervening pathways, themselves complex in terms of both number of elements and distribution, remain inaccessible for electrophysiological investigation (Barnes and Gladden, 1985; Rossignol et al., 1988). Alternatively, the study of reflexes in reduced in vitro preparations has almost invariably been performed in the absence of activity in the target motor circuitry (Burrows, 1992) and therefore under open-loop conditions that bear little functional relationship to normal rhythmic behavior in vivo. To date, the only other examples in which proprioceptive feedback to an active motor pattern generator has been successfully studied at the cellular level is the influence of stretch receptor input to the flight system of locust (Wolf and Pearson, 1987, 1988) and the walking limb of crayfish (Sillar and Skorupski, 1986; Sillar et al., 1986; Skorupski and Sillar, 1986).

\section{Parallel sensorimotor processing can reinforce or reorganize the gastric mill pattern}

Of fundamental importance to the present study was the earlier finding that active contraction of gastric mill power-stroke muscle gm1 is the effective stimulus for AGR in vivo (Combes et al., 1995a). Therefore, we were able to reproduce appropriately timed input from AGR in our in vitro experimental conditions by electrical stimulation of the receptor in time with rhythmic GM neuron bursts. Our main finding is that feedback from this single sensory neuron can have two distinctly different effects on spontaneous gastric mill network rhythmicity, inducing distinct motor patterns that occur in the intact animal (Combes et al., 1999). We have also shown that these effects are mediated by the CG and GI interneurons and that selective direct activation of one (CG alone) or both (CG and GI) interneurons closely mimics the reinforcing and reconfiguring effects of the AGR neuron itself. These results are summarized in Figure 8, which shows the functional sensorimotor circuits and resultant gastric mill output patterns in response to low and high frequencies of receptor discharge. During moderate AGR firing (Fig. $8 A$ ), the excitatory sensorimotor pathway via the $\mathrm{CG}$ neuron predominates and thereby evokes simultaneous excitation of GM and LG-MG motoneurons. Under these conditions, the spontaneous type I pattern typically observed in vitro in which medial (GM) and lateral teeth (LG-MG) power-stroke motoneurons fire in phase (Fig. 2) is promoted. During higher AGR discharge levels (Fig. 8B), both interneuronal pathways become active, and interneuron GI now inhibits the LG-MG motoneurons, permitting the GM and LPG neurons to be excited by interneuron CG. These combined effects induce a new type II gastric mill pattern in which medial tooth protractor (GM) and lateral teeth opener (LPG) motoneurons fire in phase. In functional terms, this reorganization involves a complete change in coordination (Fig. 8, ellipses) between motoneurons controlling the medial and lateral teeth subsystems and in vivo is presumably responsible for adaptive changes in masticatory teeth movements, such as those reported previously in spiny lobster (Heinzel, 1988).

\section{Comparison with other sensorimotor systems}

Studies in a variety of invertebrate and vertebrate preparations have demonstrated repeatedly the crucial role of proprioceptive feedback in adapting rhythmic motor programs to changing behavioral demands. Such influences include the regulation and reinforcement of ongoing movement amplitude and timing, and the control of phase transitions in a single movement cycle (Pearson, 1995). In this context, feedback from AGR can also participate in the entrainment and reinforcement of the gastric mill rhythm (Elson et al., 1994) by promoting GM and (indirectly) LG-MG motoneuron bursts via the excitatory CG interneuron (Simmers and Moulins, 1988a,b). However, the recruitment of the inhibitory GI interneuron and the consequent sustained reconfiguration of gastric mill motor coordination at higher rates of receptor discharge constitute a completely different sensorimotor effect that has not been demonstrated previously. Phenomenologically, this switch is analogous to the changes in interlimb coordination that, for example, permit a horse to alternate between trotting or pacing, changes that are believed to arise from different longitudinal combinations of bilaterally alternating forelimb and hindlimb locomotor programs (Pearson and Duysens, 1976).

Another reported example that resembles our results is found in Xenopus embryos (Soffe, 1993, 1997) in which mild mechanical stimulation of touch-sensitive skin sensory neurons activates the central neuronal circuit for swimming, whereas stronger stimulation recruits additional neurons, resulting in struggling. Here, however, neither the sensorimotor pathways nor the selection mechanism are completely known, and, in contrast to the gastric mill system, the different locomotor programs are triggered by brief mechanosensory stimulation rather than being continuously driven by phasic movement-related feedback.

There are also precedents for routing sensory information to a target CPG via antagonistic interneurons. In mammals and arthropods alike, multiple proprioceptive pathways, both excitatory and inhibitory, to the same functional group of leg motoneurons have been well documented (Skorupski and Bush, 1992; De Serres et al., 1995; Pearson, 1995; Leibrock et al., 1996). In these cases, the selection between different sensory pathways depends not on the activity level of the sensory neurons themselves but on the animal's behavioral state (static or locomotory) or on which phase in an individual cycle the stimulus is delivered. In mammals, input from intraoral receptors also accesses the masticatory CPG via two different interneuronal pathways, one excitatory that provides positive feedback to jaw closure motoneurons and one inhibitory that provides negative feedback and can prematurely terminate jaw closure (Rossignol et al., 1988; Appenteng, 1991). Although in this system a specific population of sensory neurons can evoke different behavioral responses via a stimulusdependent selection of different cellular pathways, again unlike our crustacean model, the selection process results in either 


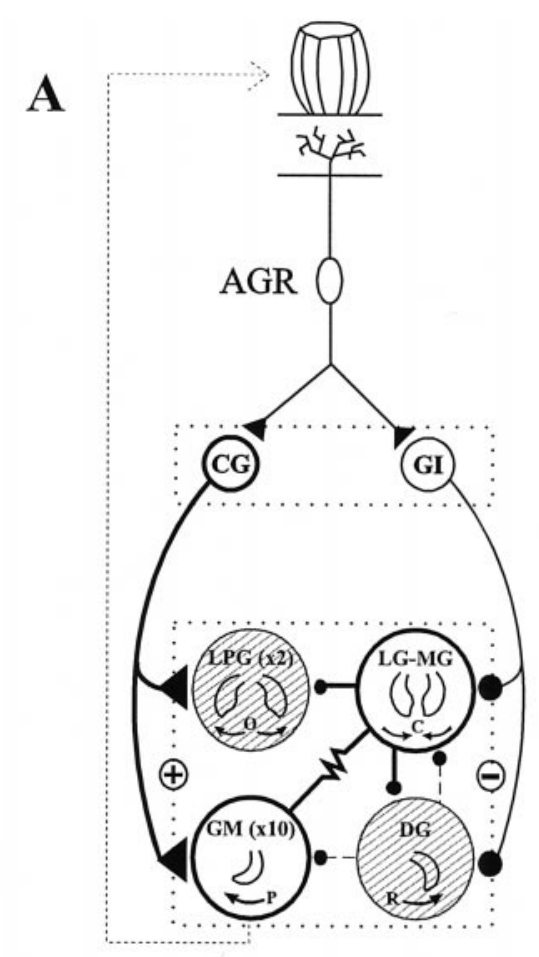

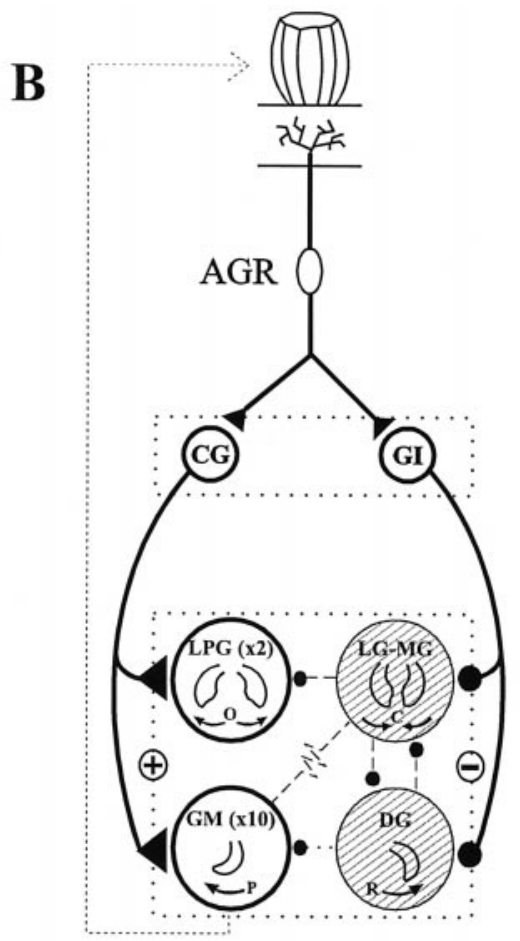

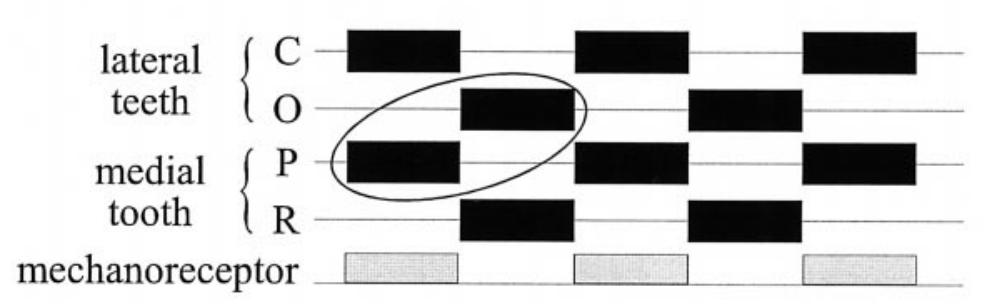

\section{LG-MG
LPG
GM
DG
AGR}

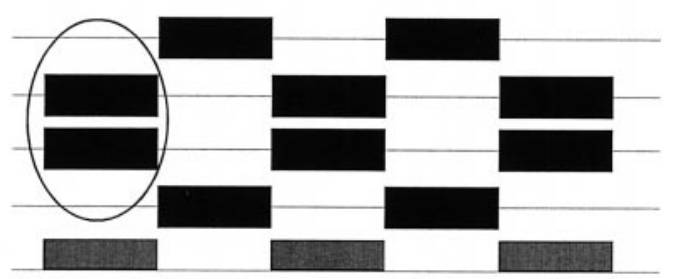

Figure 8. Schematic representation of the multiple effects of AGR on the gastric mill network and its motor pattern. During low receptor discharge $(A)$, the higher spontaneous activity of the CG interneuron ensures that its excitatory pathway predominates, thereby reinforcing the type I gastric mill pattern in which the GM and LPG neurons burst in antiphase. With intense receptor firing $(B)$, strong synaptic facilitation in the GI interneuron ensures that its inhibitory pathway becomes effective, resulting in a reconfiguration of the gastric mill pattern in which the GM and LPG neurons are now in phase with AGR firing. Open circles in gastric mill network wiring diagrams denote motoneuron subtypes that fire in phase with AGR. Hatched circles denote motoneurons that fire in antiphase with AGR. Line thickness indicates strength of corresponding synaptic pathway.

reinforcement or protective termination of a single phase of movement, not the induction of a new motor program.

\section{Mechanisms subserving sensorimotor processing: activity-dependent synaptic facilitation}

Unlike the systems described above, the relative simplicity of our preparation allowed access to the mechanisms responsible for the selection of the sensorimotor pathways on which the differential effects of AGR on the gastric mill network depend. Specifically, our results indicate that the balance of firing in the two parallel interneuronal pathways is determined by an interplay between relative differences in both intrinsic excitability and synaptic sensitivity to excitatory input from a single receptor. Whereas the CG interneuron displays high spontaneous firing rates at or near resting potential, the GI interneuron is less spontaneously active but expresses strong activity-dependent synaptic facilitation in response to AGR activity. Thus, with weak to moderate levels of receptor discharge, the higher intrinsic activity of the CG neuron ensures that this excitatory pathway is favored, whereas, at higher levels of receptor firing, the strong facilitating capability of the GI neuron ensures that the inhibitory pathway now also becomes effective. As a consequence, feedback from AGR switches from a reinforcing to a reconfiguring influence on gastric mill motor output.

Our results thus reveal a crucial role for synaptic facilitation in sensory information processing within the CNS. In the peripheral nervous system of cricket (Davis and Murphey, 1993) and lobster (Katz et al., 1993), mixed weakly and highly facilitating synapses from a common sensory input generate different temporal sequences of muscle contraction. Such short-term activitydependent synaptic facilitation is generally considered to be a presynaptic phenomenon involving calcium accumulation in input terminals (Katz and Miledi, 1968; Zucker, 1989). The differing influence of AGR on the CG and GI neurons suggests such plasticity may be differentially expressed in different postsynaptic targets of the same presynaptic neuron. Similar results have been observed in several systems. In cat, for example, the same Ia afferent makes central synapses that facilitate at some motoneurons and antifacilitate at others (Koerber and Mendel, 1991). In cricket, a similar functional segregation of target responses to a single cercal sensory neuron is believed to result from specific 
retrograde influences on presynaptic terminals by the postsynaptic elements themselves (Davis and Murphey, 1993), whereas, at the lobster neuromuscular junction, such divergent postsynaptic effects appear to be associated with morphological particularities of the presynaptic terminals (Katz et al., 1993). Whether similar specializations are implicated in the different sensitivities of the CG and GI neurons to AGR remains to be seen. An additional possibility is that more than one neurotransmitter may be selectively released at the different synaptic sites (Whim and Lloyd, 1989; Sossin et al., 1990; Blitz and Nusbaum, 1997) and/or which may be sensed by different postsynaptic receptor complements.

\section{REFERENCES}

Andersson O, Grillner S (1983) Peripheral control of the cat's step cycle. II. Entrainment of the central pattern generators for locomotion by sinusoidal hip movement during fictive locomotion. Acta Physiol Scand 118:229-239.

Appenteng K (1991) Parallel antagonistic proprioceptive pathways to jaw-elevator motoneurones. In: Studies in neuroscience, No 19, Locomotor neural mechanisms in arthropods and vertebrates (Armstrong DM, Bush MH, eds), pp 222-230. Manchester, UK: Manchester UP.

Barnes WJP, Gladden MH (1985) Feedback and motor control in invertebrates and vertebrates. London: Croom Helm.

Bässler U (1986) Afferent control of walking movements of the stick insect Cunicula impigra. II. Reflex reversal and the release of the swing phase in the restrained foreleg. J Comp Physiol 158:351-362.

Blitz DM, Nusbaum MP (1997) Distinct functions for cotransmitters mediating motor pattern selection by a modulatory projection neuron. Soc Neurosci Abstr 23:476.

Burrows M (1992) Local circuits for the control of leg movements in an insect. Trends Neurosci 15:226-232.

Combes D, Simmers J, Nonnotte L, Moulins M (1993) Tetrodotoxinsensitive dendritic spiking and control of axonal firing in a lobster mechanoreceptor neurone. J Physiol (Lond) 460:581-602.

Combes D, Simmers J, Moulins M (1995a) Structural and functional characterization of a muscle tendon proprioceptor in lobster. J Comp Neurol 363:221-234.

Combes D, Simmers J, Meyrand P, Moulins M (1995b) Motor programme switching by a lobster mechanoreceptor neurone in vitro: role of parallel interneuronal processing. J Physiol (Lond) 483P:191.

Combes D, Meyrand P, Simmers J (1999) Motor pattern specification by dual descending pathways to a lobster rhythm generating network. J Neurosci 19:3610-3619.

Davis GW, Murphey RK (1993) A role for postsynaptic neurons in determining presynaptic release properties in the cricket CNS: evidence for retrograde control of facilitation. J Neurosci 13:3827-3838.

De Serres SJ, Yang JF, Patrick SK (1995) Mechanism for reflex reversal during walking in human tibialis anterior muscle revealed by single motor unit recording. J Physiol (Lond) 188:249-258.

Elson RC, Panchin, YV, Arshavsky, YI, Selverston AI (1994) Multiple effects of an identified proprioceptor upon gastric pattern generation in spiny lobsters. J Comp Physiol [A] 174:317-329.

Getting PA, Dekin MS (1985) Tritonia swimming. A model system for integration within rhythmic motor systems. In: Model neural networks and behavior (Selverston AI, ed), pp 3-20. New York: Plenum.

Grillner S, McClellan A, Perret C (1981) Entrainment of the spinal pattern generators for swimming by mechanosensitive elements in the lamprey spinal cord in vitro. Brain Res 217:380-386.

Harris-Warrick RM, Marder E, Selverston AI, Moulins M (1992) Dynamic biological networks: the stomatogastric nervous system. Cambridge, MA: MIT.

Heinzel HG (1988) Gastric mill activity in the lobster. I. Spontaneous modes of chewing. J Neurophysiol 59:528-550.

Katz B, Miledi R (1968) The role of calcium in neuromuscular facilitation. J Physiol (Lond) 195:481-492.
Katz PS, Kirk MD, Govind CK (1993) Facilitation and depression at different branches of the same motor axon: evidence for presynaptic differences in release. J Neurosci 13:3075-3089.

Koerber RH, Mendel LM (1991) Modulation of synaptic transmission at Ia-afferent fiber connections on motor neurons during high frequency stimulation: role of the post synaptic target. J Neurophysiol 65:590-597.

Leibrock CS, Marchand AR, Barnes WJP, Clarac F (1996) Synaptic connections of the cuticular stress detectors in crayfish: mono- and polysynaptic reflexes and the entrainment of fictive locomotion in an in vitro preparation. J Comp Physiol [A] 178:711-725.

Miller JP, Selverston AI (1979) Rapid killing of single neurons by irradiation of intracellular injected dye. Science 206:702-704.

Pearson KG (1995) Proprioceptive regulation of locomotion. Curr Opin Neurobiol 5:786-791.

Pearson KG, Duysens JD (1976) Function of segmental reflexes in the control of stepping in cockroaches and cats. In: Neural control of locomotion (Herman RM, Grillner S, Stein PSG, Stuart DG, eds), pp 519-538. New York: Plenum.

Reye DN, Pearson KG (1988) Entrainment of the locust central flight oscillator by wing stretch receptor stimulation. J Comp Physiol 162:77-89.

Rossignol S, Lund JP, Drew T (1988) The role of sensory inputs in regulating patterns of rhythmical movements in higher vertebrates. In: Neural control of rhythmic movements in vertebrates (Cohen $\mathrm{AH}$, Rossignol S, Grillner S, eds), pp 201-283. New York: Wiley.

Selverston AI, Moulins M (1987) The crustacean stomatogastric system. Heidelberg: Springer.

Sherrington CS (1917) Reflexes elicitable in the cat from pinna, vibrissae and jaws. J Physiol (Lond) 51:404-431.

Sillar KT, Skorupski P (1986) Central input to primary afferent neurons in crayfish, Pacifastacus leniusculus, is correlated with rhythmic motor output of thoracic ganglia. J Neurophysiol 55:678-688.

Sillar KT, Skorupski P, Elson RC, Bush BMH (1986) Two identified afferent neurones entrain a central locomotor rhythm generator. $\mathrm{Na}-$ ture 323:440-443.

Simmers J, Moulins M (1988a) A disynaptic sensorimotor pathway in the lobster stomatogastric system. J Neurophysiol 59:740-756.

Simmers J, Moulins M (1988b) Nonlinear interneuronal properties underlie integrative flexibility in a lobster disynaptic sensorimotor pathway. J Neurophysiol 59:757-777.

Skorupski P, Bush BMH (1992) Parallel reflex and central control of promotor and receptor motoneurons in crayfish. Proc R Soc Lond B Biol Sci 249:7-12.

Skorupski P, Sillar KT (1986) Phase-dependent reversal of reflexes mediated by the thoraco-coxal muscle receptor organ in the crayfish, Pacifastacus leniusculus. J Neurophysiol 55:689-695.

Soffe SR (1993) Two distinct rhythmic motor patterns are driven by common premotor and motor neurons in a simple vertebrate spinal cord. J Neurosci 13:4456-4469.

Soffe SR (1997) The pattern of sensory discharge can determine the motor response in young Xenopus tadpoles. J Comp Physiol [A] 180:711-715

Sossin WS, Sweet-Cordero A, Scheller RH (1990) Dale's hypothesis revisited: different neuropeptides derived from a common prohormone are targeted to different processes. Proc Natl Acad Sci USA 87:4845-4848.

Whim MD, Lloyd PE (1989) Frequency-dependent release of peptide cotransmitters from identified cholinergic motor neurons in Aplysia. Proc Natl Acad Sci USA 86:9034-9038.

Wolf H, Pearson KG (1987) Intracellular recordings from interneurons and motoneurons in intact flying locusts. J Neurosci Methods 21:345-354.

Wolf H, Pearson KG (1988) Proprioceptive input patterns elevator activity in the locust flight system. J Neurophysiol 59:1831-1853.

Zucker RS (1989) Short-term synaptic plasticity. Annu Rev Neurosci 12:13-31. 